\title{
Modelling the movement of interacting cell populations
}

\author{
Kevin J. Painter*, Jonathan A. Sherratt \\ Department of Mathematics, Centre for Theoretical Modelling in Medicine, Heriot-Watt University, Edinburgh EH14 4AS, UK
}

Received 7 April 2003; received in revised form 20 June 2003; accepted 23 June 2003

\begin{abstract}
Mathematical modelling of cell movement has traditionally focussed on a single population of cells, often moving in response to various chemical and environmental cues. In this paper, we consider models for movement in two or more interacting cell populations. We begin by discussing intuitive ideas underlying the extension of models for a single-cell population to two interacting populations. We then consider more formal model development using transition probability methods, and we discuss how the same equations can be obtained as the limiting form of a velocity-jump process. We illustrate the models we have developed via two examples. The first of these is a generic model for competing cell populations, and the second concerns aggregation in cell populations moving in response to chemical gradients.
\end{abstract}

(C) 2003 Elsevier Ltd. All rights reserved.

Keywords: Cell migration; Tissue; Travelling waves; Chemotaxis

\section{Introduction}

Mathematical modelling of cell movement has a long and successful history. A key early paper was that of Keller and Segel (1970), who developed a partial differential equation model to study the biochemical regulation of bacterial movement. Their highly influential equations have been the basis for models of phenomena as diverse as slime mould aggregation (Höfer et al., 1995), tumour angiogenesis (Chaplain and Stuart, 1993), primitive streak formation (Painter et al., 2000) and wound repair (Pettet et al., 1996). In the 1980 s, a separate area of research developed, in which models were proposed for the movement of isolated single cells. This work was lead initially by Oster (Oster, 1984; Oster and Perelson, 1985), and has subsequently been developed by a range of authors (Bottino and Fauci, 1998; Bottino et al., 2002). In principle, these two modelling approaches should converge to give a macroscopic model that reflects in detail microscopic cell behaviour. There have been a number of recent papers in this direction; e.g. Othmer and Stevens (1997)

*Corresponding author. Tel.: + 44-131-451-8005; fax: + 44-131-4513249 .

E-mail addresses: painter@ma.hw.ac.uk (K.J. Painter), jas@ma. hw.ac.uk (J.A. Sherratt). and Schnitzer et al. (1990) explore the derivation of macroscopic equations for chemotaxis from a biased random walk incorporating detailed microscopic behaviour rules, while Dallon and Othmer (1997) considers the individual movement of discrete cells in a continuous chemical field.

In this paper, we consider modelling a different aspect of cell movement, namely behaviour in a mixture of two interacting cell populations. This is a very common scenario in physiological contexts. For example, tumour cells typically move through and interact with the surrounding population of untransformed cells, and wound healing in the corneal epithelium depends on the migration into the wound of interacting subpopulations of epithelial cells. In development, the reorganization of undifferentiated cells of the early embryo into multipletissue types during gastrulation requires the coordinated movement of distinct subpopulations.

Despite these and other important examples, very little previous work has been done in this area. Most models of interacting cell populations include movement very simply, via independent linear diffusion of each population (Sherratt and Nowak, 1992; Pettet et al., 1996; Gaffney et al., 1999). This will typically be appropriate when the individual cells are widely separated. However, when cells are close enough for regular contacts, those of one type will inevitably 
influence the movement of the other cell population. The objective of this paper is to consider how this can be best reflected in partial differential equation models. In Section 2 we discuss intuitive ideas behind models for interacting cell populations, and in Section 3 we develop model equations using a space-jump approach. In Section 4 we consider suitable functional forms for motility coefficients and their biological implications, and in Section 5 we discuss the possibility of deriving the equations using the alternative velocity-jump formalism. Finally, in Sections 6 and 7 we present two examples of the application of our model to particular types of cell behaviour.

\section{Intuitive considerations}

\subsection{One-cell population}

When individual cells in a population are widely separated, they will have little direct interaction. Therefore, one can reasonably assume that in the absence of external cues biasing their motion, each cell will undergo an unbiased random walk. At the population level, this implies that diffusion is the appropriate mathematical model. As the cell density increases, individual cells will interact more often, with an inevitable effect on motility. However, in the absence of external cues their motion will remain unbiased, so that one expects a diffusion-like term to still be appropriate. This suggests a model with a nonlinear diffusion coefficient, dependent on cell density. Denoting this density by $u(\underline{x}, t)$, the appropriate model is thus

$\partial u / \partial t=D \nabla \cdot[Q(u) \nabla u]$.

(We omit any cell kinetic terms at this stage to focus on motility). It is most convenient to define $D$ as the diffusion coefficient when the individual cells are widely separated; this implies that $Q(0)=1$. As the cell density goes up, we expect the diffusion coefficient to decrease as a result of cell-cell interactions, so that $Q($.$) is$ decreasing.

\subsection{Multiple cell populations}

In the case of interacting cell populations, modelling is again straightforward when individual cells are widely separated. If there are no external cues biasing motion, we can again reasonably assume that each cell undergoes an unbiased random walk, which means that at the population level, the appropriate model is linear diffusion for each population. As the cell density increases, cell-cell interactions will again affect movement. The simplest case to consider is when the two-cell populations have identical movement properties. If we take $u(\underline{x}, t)$ and $v(\underline{x}, t)$ to be the densities of the two cell types, then the total cell density $u+v$ will satisfy the nonlinear diffusion equation (1)

$\partial(u+v) / \partial t=D \nabla \cdot[Q(u+v) \nabla(u+v)]$.

To separate this into equations for $u$ and for $v$, it is helpful to reconsider the interpretation of the nonlinear diffusion. At a macroscopic level, Eq. (1) implies that cells move down gradients of their own density, at a rate that depends on that density via the function $Q($.$) . In the$ case of two-cell populations, cells may move in response to either the gradient in their own density, or the gradient in total cell density. This will depend on the details of cell behaviour. In general, we can divide the term $Q(u+v) \nabla(u+v)$ in (2) into two parts: $A(u+v)$ $\nabla(u+v)$ which is the movement of cells down gradients of their own density, and $B(u+v) \nabla(u+v)$ which is the movement of cells down gradients of total cell density. Here $A+B=Q$. The first term implies a contribution $A(u+v) \nabla u$ to the movement of the $u$ cell population, and $A(u+v) \nabla v$ to the movement of the $v$ population. The second term will divide between the two populations simply according to the ratio of their densities, giving contributions $u /(u+v) B(u+v) \nabla(u+v)$ and $v /(u+v) B(u+v) \nabla(u+v)$ to the movement of the two populations. Therefore, the overall equations governing cell movement are

$$
\begin{aligned}
& \frac{\partial u}{\partial t}=D \nabla \cdot\left[A(u+v) \nabla u+\frac{u}{u+v} B(u+v) \nabla(u+v)\right], \\
& \frac{\partial v}{\partial t}=D \nabla \cdot\left[A(u+v) \nabla v+\frac{v}{u+v} B(u+v) \nabla(u+v)\right] .
\end{aligned}
$$

The forms of the functions $A($.$) and B($.$) will depend on$ the details of cell behaviour, and some potential functions are discussed below. Intuitively we expect that, like the function $Q($.) discussed above, $A($.$) and B()$. will be decreasing functions.

\section{Jump probability calculations}

Continuous equations for cell movement are commonly derived from two classes of movement, coined "velocity-jump" and "space-jump" in Othmer et al. (1988). The former models movement as periods of smooth motion punctuated by reorientations, while the latter assumes a sequence of discrete jumps in space. Both approaches enable continuum equations to be derived in appropriate limits, but this process is more straightforward for the space-jump formalism. Therefore we consider this first, and return briefly to the velocity-jump approach in Section 5.

To derive our model for movement, we employ the method of Othmer and Stevens [for more details, see Othmer and Stevens (1997) and the reference therein], where a master equation for a continuous-time, 
discrete-space random walk on a one-dimensional equidistant lattice is considered. We define $u_{i}(t)$ to be the probability of a cell to be at $i \in \mathbf{Z}$ at time $t$, conditioned on beginning at $i=0$ at $t=0$. We assume this evolves according to the continuous-time discretespace equation:

$\frac{\partial u_{i}}{\partial t}=\mathscr{T}_{i-1}^{+} u_{i-1}+\mathscr{T}_{i+1}^{-} u_{i+1}-\left(\mathscr{T}_{i}^{+}+\mathscr{T}_{i}^{-}\right) u_{i}$.

In the above, $\mathscr{T}_{i}^{ \pm}(\cdot)$ defines the transitional probabilities per unit time of a one-step jump to $i \pm 1$. The above model simply describes the changing cell numbers as individuals enter or leave a site, and herein we shall equate the probability distribution with the cell density.

The simplest assumption is that the jump probability is equal in either direction and uniform across the lattice, i.e. $\mathscr{T}_{i}^{ \pm}=\alpha$, constant. Thus,

$\frac{\partial u_{i}}{\partial t}=\alpha\left(u_{i-1}+u_{i+1}-2 u_{i}\right)$.

We set $x=i h$, reinterpret $x$ as a continuous variable and extend the definition of $u_{i}$ accordingly. By introducing a scaling of the transition probabilities such that $T^{ \pm}=$ $\lambda T^{ \pm}$, and expanding the right-hand side as Taylors equations in functions of $x$ we obtain

$\frac{\partial p}{\partial t}=\lambda h^{2} \alpha \frac{\partial^{2} p}{\partial x^{2}}+\mathcal{O}\left(h^{4}\right)$.

As the spatial scale $h$ is changed, the transitional probabilities of a jump must be changed accordingly. Thus we assume that the following limit exists:

$\lim _{\substack{h \rightarrow 0 \\ \lambda \rightarrow \infty}} \lambda h^{2}=$ constant $\equiv c$,

and one derives the diffusion equation:

$\frac{\partial u}{\partial t}=D_{u} \frac{\partial^{2} u}{\partial x^{2}}$

where $D_{u}=c \alpha$ is a constant.

The assumption of a uniform isotropic jump probability is inappropriate for most cell populations, whose movement is strongly influenced by interaction with environmental signals (e.g. chemicals, gravity, light), or contact with other cells (e.g. contact inhibition, adhesion). This can be incorporated into the above model by assuming a dependence in the jump probabilities. This was studied in the context of chemosensitive movement in Othmer and Stevens (1997), where different macroscopic (PDE) models were derived, depending on the local strategy for environment sensing.

\subsection{Strategies for sensing}

Here, we develop a general model for the biased movement of a cell on a lattice, where the jump probabilities depend on a variety of environmental factors (e.g. other cell populations or chemicals). For present, we keep the approach general, but later we shall explicitly consider the case where cell movement depends on the total cell density. We denote by $E$ the matrix of the environmental cues, where $E_{j, i}$ indicates the density or concentration of the $j$-th factor at lattice site $i$. For example, for the situation discussed in Section $2, j$ would only take the value 1 , with $E_{1, i}$ being the total cell density at site $i$.

We consider four sensing strategies:

1. Strictly local: information only at the present position is considered.

2. Neighbour based: considers information at the target jump site.

3. Local average: considers the average of the information between the particles present and target site.

4. Gradient: considers the local difference in information between the target and local site.

Of course, this by no means characterises all the strategies a cell may employ. In particular, other mechanisms may incorporate longer ranging information, for example if a cell extends filopodia out into its environment.

1. Strictly local models: For strictly local rules, we choose $\mathscr{T}_{i}^{+}=\mathscr{T}_{i}^{-}=f\left(E_{i}\right)$ where $E_{i}$ represents the information at $i$. The Master equation becomes

$\frac{\partial u_{i}}{\partial t}=f\left(E_{i+1}\right) u_{i+1}-2 f\left(E_{i}\right) u_{i}+f\left(E_{i-1}\right) u_{i-1}$.

Under the appropriate scaling, the following PDE is derived:

$\frac{\partial u}{\partial t}=D \frac{\partial^{2}}{\partial x^{2}}(f(E) u)$.

2. Neighbour based: For neighbour-based rules we assume $\mathscr{T}_{i}^{+}=g\left(E_{i \pm 1}\right)$, resulting in the following Master equation:

$\frac{\partial u_{i}}{\partial t}=g\left(E_{i}\right)\left(u_{i+1}+u_{i-1}\right)-u_{i}\left(g\left(E_{i+1}\right)+g\left(E_{i-1}\right)\right)$,

and in the PDE limit we derive

$\frac{\partial u}{\partial t}=\frac{\partial}{\partial x}\left[g(E) \frac{\partial u}{\partial x}-u \frac{\partial g(E)}{\partial x}\right]$.

Phenomenologically, for decreasing $g$, this models processes such as "space-limitation", in which a cell is only able to move into a neighbouring site if there is sufficient space available.

3. Local average: We assume $\mathscr{T}_{i}^{ \pm}=h\left(\left(E_{i \pm 1}+E_{i}\right) / 2\right)$. If substituted into the master equation we obtain

$\frac{\partial u_{i}}{\partial t}=h\left(\frac{E_{i+1}+E_{i}}{2}\right)\left(u_{i+1}-u_{i}\right)-h\left(\frac{E_{i}+E_{i-1}}{2}\right)\left(u_{i}-u_{i-1}\right)$

and the PDE equation is derived as

$\frac{\partial u}{\partial t}=\frac{\partial}{\partial x}\left(h(E) \frac{\partial u}{\partial x}\right)$. 
4. Gradient-based models: The gradient-based model assumes transitional probabilities of the form

$\mathscr{T}_{i}^{ \pm}=\alpha+\beta\left(\tau\left(E_{i}\right)-\tau\left(E_{i \pm 1}\right)\right)$

and the resulting PDE is

$\frac{\partial u}{\partial t}=\frac{\partial}{\partial x}\left[D \frac{\partial u}{\partial x}+u \frac{\mathrm{d} \tau}{\mathrm{d} E} \frac{\mathrm{d} E}{\mathrm{~d} x}\right]$.

The above equation has been employed extensively to model tactic responses in cell populations, such as chemotaxis, haptotaxis or phototaxis.

5. Combined models: Since cell movement involves the processing of multiple signals, each of which may act on the cell in different ways, a combination of the above strategies may be necessary to most accurately reflect cell movement. While the mathematical form of the equations quickly becomes complex, the derivation is relatively straightforward. For example, combining each of the local, neighbour and gradient-based models above gives

$\mathscr{T}_{i}^{ \pm}=f\left(E_{i}\right) g\left(E_{i} \pm 1\right)\left(\alpha+\beta\left(\tau\left(E_{i}\right)-\tau\left(E_{i \pm 1}\right)\right)\right)$.

The PDE corresponding to this combined movement rule is

$$
\begin{aligned}
\frac{\partial u}{\partial t}= & \frac{\partial}{\partial x}\left[g(E) \frac{\partial}{\partial x}(f(E) u)-f(E) u \frac{\partial g(E)}{\partial x}\right. \\
& \left.+u g(E) f(E) \frac{\mathrm{d} \tau}{\mathrm{d} E} \frac{\mathrm{d} E}{\mathrm{~d} x}\right] .
\end{aligned}
$$

\section{Forms of motility functions for interacting cell populations}

We now consider the case outlined in Section 2, where two cell populations $u$ and $v$ interact via the movement depending on the total cell density, $w=u+v$. Cases (1)(4) above thus give rise to the following equations for the dynamics of the $u$ population:

$$
\begin{aligned}
& \frac{\partial u}{\partial t}=\frac{\partial}{\partial x}\left(f(w) \frac{\partial u}{\partial x}+u \frac{\partial f(w)}{\partial w} \frac{\partial w}{\partial x}\right), \\
& \frac{\partial u}{\partial t}=\frac{\partial}{\partial x}\left(g(w) \frac{\partial u}{\partial x}-u \frac{\partial g(w)}{\partial w} \frac{\partial w}{\partial x}\right), \\
& \frac{\partial u}{\partial t}=\frac{\partial}{\partial x}\left(h(w) \frac{\partial u}{\partial x}\right), \\
& \frac{\partial u}{\partial t}=\frac{\partial}{\partial x}\left(D \frac{\partial u}{\partial x}+u \chi(w) \frac{\partial w}{\partial x}\right) .
\end{aligned}
$$

In the above, $\chi(w)=c \mathrm{~d} \tau / \mathrm{d} w$. Clearly, all of the above models fit into the intuitively derived forms, Eqs. (3).

How could the total cell density affect the movement properties of the cells? Here we shall restrict our attention to those mechanisms which may lead to dispersal of the population (i.e. we ignore adhesive
Table 1

Table illustrating how the terms of the generic model vary for different local rules

\begin{tabular}{llll}
\hline Scheme & Example function & $A(w)$ & $B(w)$ \\
\hline Local & $f=\gamma w$ & $\gamma w$ & $\gamma w$ \\
Neighbour & $g=1-w / T$ & $1-w / T$ & $w / T$ \\
Average & $h=1-w / T$ & $1-w / T$ & 0 \\
Gradient & $\chi>0, D=0$ & 0 & $\chi w$ \\
\hline
\end{tabular}

$\gamma, T, \chi$ and $D$ are all assumed constant.

type processes). We consider the following general mechanisms:

- "Population pressure": We assume that a high cell density results in increased probability of a cell being "pushed" from a site, for example due to the pressure exerted by neighbouring cells. This might be achieved phenomenologically using the strictly local formulation and $f(w)$ increasing.

- "Limited space": Here we assume that no more cells can enter a site above a total cell density. In the context of the local rules, above, this may be achieved with either the neighbour or average-based model, and choosing $g(w)$ or $h(w)$ such that there exists some $T$ for which $g(T)=0$ when $w=T$.

- "Gradient" detection: Cells may detect and respond to a local gradient in the cell density, in which case we assume the gradient model. To ensure that cells move down gradients in the total density (i.e. homogenizing) we require $\chi(w)>0$. If we assumed further that movement occurs only when a gradient is detected then we would choose $D=0$.

For simple choices of the functional forms, the above mechanisms give rise to the forms for $A$ and $B$ given in Table 1. Note that for the above mechanisms $A$ and $B$ are $\geqslant 0$ (providing $w \leqslant T$ ). Thus we expect the equations to be well-defined.

\subsection{Cell-marking experiments}

We first illustrate the effect cell-cell interactions by comparing the movements of a cell population for a model in which this is incorporated (we use the "neighbour" scheme form of Table 1) to the independent movement scenario [i.e. $A \equiv 1, B \equiv 0$ in Eq. (3)]. To monitor the mixing, we assume a hypothetical experimental set-up in which a population of identical cells is seeded at high density at one end and a low density at the other (see schematic in Fig. 1). A proportion of the cells are marked in a manner such that those cells form a homogeneous distribution. Under independent movement, while the total cell density evolves to a homogeneous movement, no net movement is observed in the marked subpopulation, Fig. 1(a). When interaction is included, however, although the total cell density evolution is the identical, clear 

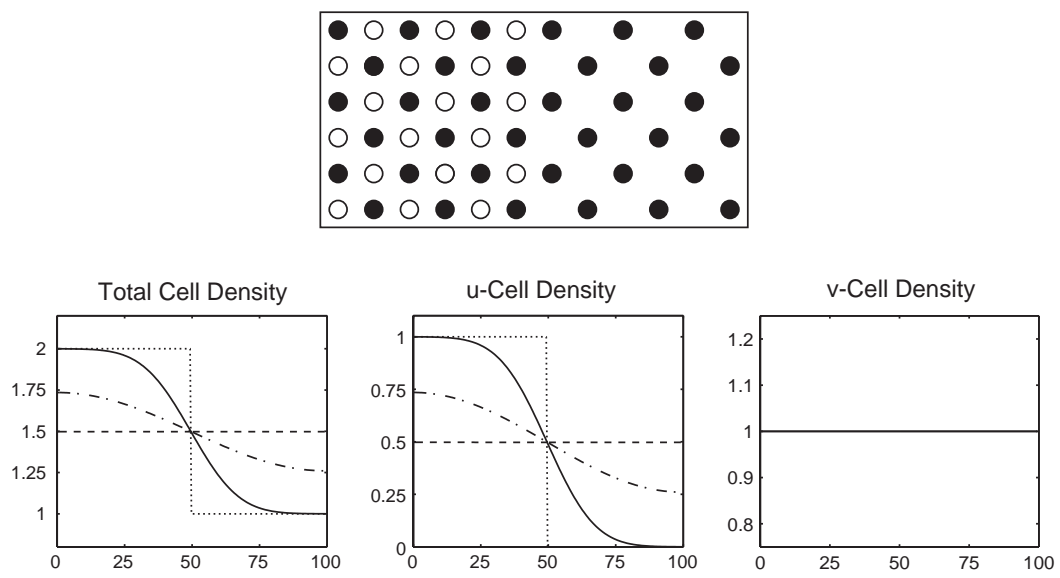

(a)
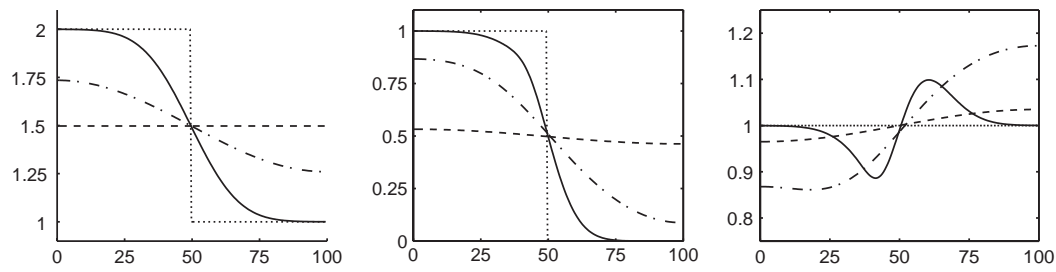

(b)

Fig. 1. Comparison of independent (i.e. $A \equiv 1, B \equiv 0$ ) and interacting scenarios. Top row; schematic showing distribution of marked (black, $v$ )/ unmarked (white cells, $u$ ). (a) Evolution for independent diffusion $(A=1, B=0)$. (b) Evolution for interacting scenario (choosing "neighbour" scheme from Table 1, with $T=2$ ). In both sets, time shown are $t=0$ (dotted line), $t=100$ (solid), $t=1000$ (dot-dash) and $t=10000$ (dashed).

distinction can be seen in the movements of marked/ unmarked subpopulations, with the marked cells experiencing a flux due to the total cell density gradient, Fig. 1(b). We note that when the cells are seeded at much lower densities (i.e. such that the total cell density $\ll T$ ), this effect is less pronounced: away from the maximum packing, cell contact is less frequent, and we are closer to the independent movement scenario.

We now address the question of whether differences in the local mechanisms considered in Table 1 can be understood through the behaviour of the macroscopic equations by considering two hypothetical experimental set-ups. In the first, Fig. 2(a), a population of (motile) identical cells are seeded with a gradient in the cell density. A fraction of these cells are labelled with a marker such that the density of labelled cells is uniform. In the second set-up, Fig. 2(b), the population is initially seeded at a uniform density, while the marked fraction is set in a graded manner.

In Fig. 2 we summarize the results of the experiments. The local and neighbour-based rules show very similar behaviour, as may be expected by the comparatively close forms of the PDE, see Fig. 2. For type I experiments, $u$ has a uniform distribution, yet the total density varies. The resulting flux from the total density induces a net migration of $u$ cells down this gradient, before both populations eventually become homogeneously distributed. For type II experiments, the total cell density is constant; and thus each population moves down its own gradient. A difference can be seen when looking at the behaviour at the maximum packing density, where the limited-space model predicts that no movement can occur and cells are fixed in the initial configuration.

In contrast, if there is no flux contribution due to the total cell gradient (i.e. average based model), differences in behaviour can clearly be seen in the type I experiment, Fig. 2. Despite a gradient in total cell density, $u$, the cells remain homogeneously distributed. The type II set-up shows the same behaviour as described above.

The gradient system can also be easily distinguished. Here, cells respond only to the total density gradient. Thus for type II experiments, no movement occurs, and the cell populations remain in the initial configuration. For type I experiments, mixing occurs until the total cell density becomes homogeneous, though the subpopulations themselves may remain inhomogeneously mixed.

\section{Velocity-jump calculations}

Before presenting the application of our equations to specific situations, we consider briefly the possibility of deriving model equations using the "velocity-jump" approach (Othmer et al., 1988). This assumes that cells undergo periods of smooth motion punctuated by 

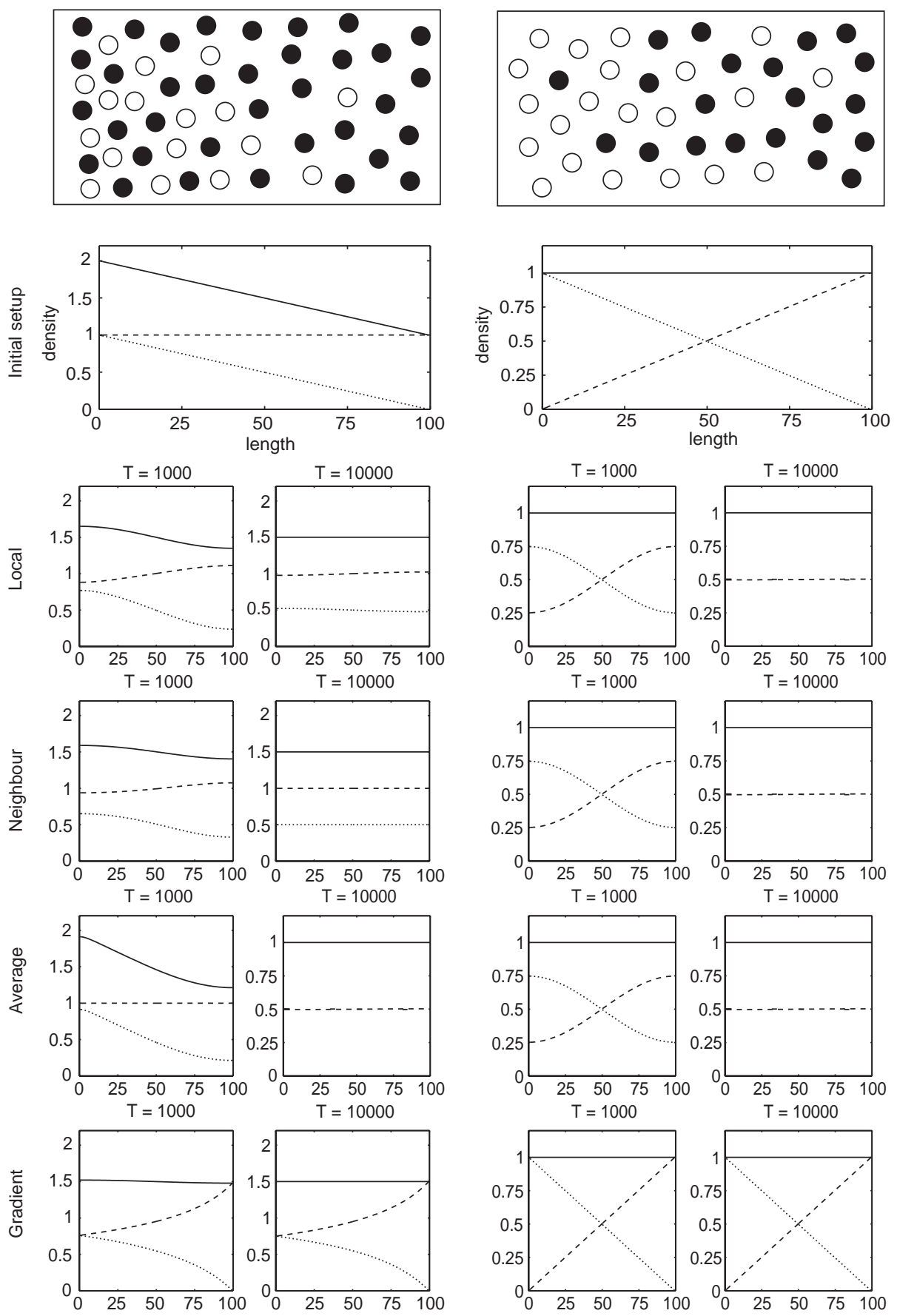

Fig. 2. Comparison between the various movement rules for two hypothetical experiment set ups. Top rows: Schematic showing distribution of marked (black)/unmarked (white) cells for the experiments. Bottom rows: Snapshots in the time evolution for different schemes, with $A(w)$ and $B(w)$ forms corresponding to those given in Table 1, showing total cell density (solid), marked cell density (dashed) and unmarked cell density (dotted). The $T=10000$ plots indicate the steady-state solutions. Simulations use $T=2, \gamma=1, \chi=1$ where appropriate.

reorientations. In one space dimension, this means that movement can be characterized by three parameters: the cell speed ( $s$ say), and the rates at which cells moving to the left and right change direction $(\lambda \pm \delta$, say). Any difference between these last two parameters (i.e. $\delta \neq 0$ ) indicates a directed component to the movement.

Hillen and coworkers (Hillen, 2002) have studied in detail the development of partial differential equations to describe velocity-jump processes. In particular, Hillen and Stevens $(2000)^{1}$ showed that in the limit of high cell

\footnotetext{
${ }^{1}$ The derivation of the parabolic limit in Hillen and Stevens (2000) assumes that the quantity $2 \lambda-(1 / s) \partial s / \partial t$ is independent of $x$. More generally, although the underlying telegraph equation is different, the same parabolic equation emerges in the limit of high cell speed and turning rate (Hillen, personal communication).
} 
speed and high turning rate with $s^{2} / \lambda$ remaining finite, the underlying telegraph equation approaches a parabolic limit, given by

$\frac{\partial u}{\partial t}=\frac{\partial}{\partial x}\left[\frac{s^{3}}{2 s \lambda-\partial s / \partial t} \frac{\partial u}{\partial x}+\frac{s^{2}(\partial s / \partial x+2 \delta)}{2 s \lambda-\partial s / \partial t} u\right]$.

In applications to interacting cell populations, $s, \lambda$ and $\delta$ will be functions of total cell density $w$ and its derivatives. For simplicity we assume that they depend only on $w, w_{x}$ and $w_{t}$ (but not higher derivatives). In this case Eq. (11) has form (3a) provided that $s, \lambda$ and $\delta$ have the following forms:

$s=s(w)$ an arbitrary function,

$\lambda=\frac{1}{2}\left[\frac{s(w)^{2}}{A(w)}+\frac{s^{\prime}(w)}{s(w)} \frac{\partial w}{\partial t}\right]$,

$\omega=\frac{1}{2}\left[\frac{B(w) s(w)}{w A(w)}-s^{\prime}(w) \frac{\partial w}{\partial x}\right]$.

Thus the mean turning rate depends on both the total cell density and its rate of change, while the difference in left and right turning rates depends on the total density and its spatial gradient.

\section{Example 1: Competing cell fronts}

A useful illustration of the movement terms derived above is given by considering two cell populations whose kinetics are of competition type. This may be competition for a nutrient, or simply for physical space.
We retain our assumption that the two populations have identical movement properties, but we assume that the $v$ cells have a competitive advantage over the $u$ cells. On a one-dimensional domain, the appropriate model equations for this situation are

$$
\begin{aligned}
\frac{\partial u}{\partial t}= & D \frac{\partial}{\partial x}\left[A(u+v) \frac{\partial u}{\partial x}+\frac{u}{u+v} B(u+v) \frac{\partial(u+v)}{\partial x}\right] \\
& +u(1-u-v), \\
\frac{\partial v}{\partial t}= & D \frac{\partial}{\partial x}\left[A(u+v) \frac{\partial v}{\partial x}+\frac{v}{u+v} B(u+v) \frac{\partial(u+v)}{\partial x}\right] \\
& +v(\gamma-u-v),
\end{aligned}
$$

where $\gamma>1$ reflects the competitive advantage of the $v$ cells. A specific instance to which this model could be applied is early tumour growth. Here $u$ and $v$ would be the density of untransformed and tumour cells, respectively; $\gamma$ would represent the proliferative advantage given by an oncogenic mutation.

Fig. 3 illustrates a typical solution of Eq. (12) for $A$ and $B$ determined using the local and neighbour-based functions from Table 1. We use initial conditions consisting of uniform densities of the two-cell populations in adjacent regions of space. This initial solution evolves into an advancing wave front in $v$ and a receding front in $u$, reflecting the competitive advantage of the $v$ population. For comparison, we also show in Fig. 3 the solution of Eq. (12) in the case of cell populations which undergo independent linear diffusion, which is given by setting $A \equiv 1$ and $B \equiv 0$. The qualitative form of the
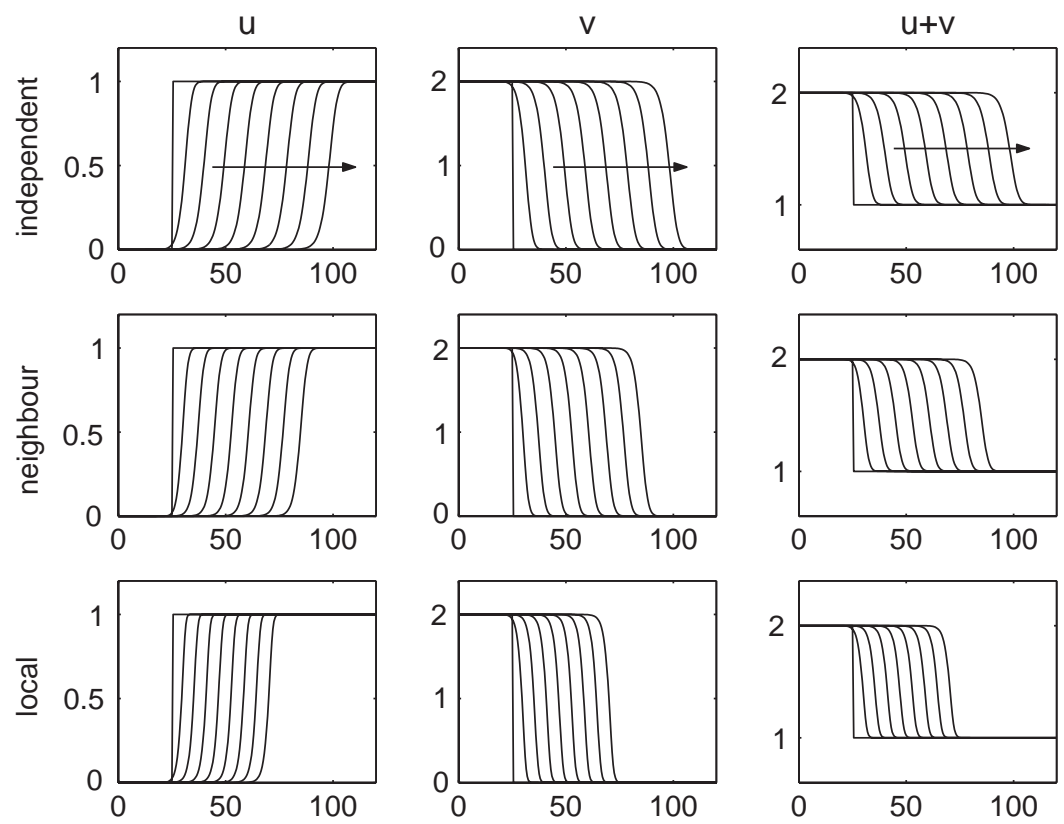

Fig. 3. Travelling wave solutions for different interactions in the two-cell competition model. Top row: independent linear diffusion $(A=1, B=0)$; centre row: "neighbour-rule", $(A=1-(u+v) / 3, B=(u+v) / 3)$; bottom row: "local-rules", $(A=(u+v) / 3, B=(u+v) / 3)$. Numerical calculation indicated waves speeds of $2,1.63$ and 1.15 respectively, confirming validity of the analytical expression. Other parameters were set at $D=1, \gamma=2$ and wave profiles are plotted at time intervals of 2 . 
solution is the same, but the speed of the wave front is noticeably higher in this case; this reflects the inhibitory effect of cell-cell interactions in cell movement.

A simple analytical argument gives an indication of the difference in wave speed in the cases shown in Fig. 3. Following a standard approach for travelling wave problems of this kind, we linearize Eq. (12) about the steady-state $v=0, u=1$, which is the limiting solution ahead of the wave. The linearized $v$ equation decouples, to give

$\frac{\partial v}{\partial t}=\frac{\partial}{\partial x}\left[A(1) \frac{\partial v}{\partial x}\right]+(\gamma-1) v+O\left(v^{2}\right)$.

By analogy with standard theory for scalar equations such as the Fisher equation, we thus expect the wave speed to be $2[A(1)(\gamma-1)]^{1 / 2}$. This formula is confirmed by numerical simulation, and indicates that the ratio of the speeds between the independent and non-independent cases in Fig. 3 is $\sqrt{A(1)}$. Note that this relation does not apply in the special case $A \equiv 0$; calculation of the wave speed is more complicated in this case, and is discussed in detail in Sherratt (2000).

\section{Example 2. Aggregation in tissues}

As a second example, we explore how the interactions in movement may affect processes of aggregation. Chemotaxis is employed by both bacteria and eukaryotic cells for directed movement and organization: for example, the aggregation of Dictyosytelium cells under starvation conditions is initiated by chemotactic movement up gradients of self-produced cAMP chemical waves, while in embryonic development guided cell movement is essential in many processes of spatial patterning and morphogenesis.

The majority of continuous treatments of chemotaxis have ignored how the complexity of the tissue environment may influence the movement dynamics. We use the framework here to address some of these issues.

The most commonly employed models for chemotaxis are based on the PDE systems proposed in Keller and Segel (1971):

$\frac{\partial u}{\partial t}=D_{u} \nabla^{2} u-\nabla \cdot(u \chi(c) \nabla c)+f(u, c)$,

$\frac{\partial c}{\partial t}=D_{c} \nabla^{2} c+g(u, c)$

where $u$ and $c$ represent cell density/chemoattractant concentrations. The function $\chi(c)$ is commonly referred to as the chemotactic sensitivity function. This system has been studied extensively, in particular for its ability to exhibit pattern formation/self-organization under suitable chemical kinetics. For example, for the choice of $f(u, c)=0, g(u, c)=\gamma u-\delta c$ and $\chi$ sufficiently strong, an initially homogeneous cell distribution forms a spatial pattern of cell aggregations. This is intuitively understood through the self-reinforcing mechanism in which cells move up gradients of a chemical they are secreting. Variations of the model have been used to model a variety of processes of aggregation in bacteria populations (e.g. Tyson et al., 1999) or development (e.g. Painter et al., 2000).

Of course, the above formulation also assumes independent movement by the cells, and thus does not realistically describe the behaviour of cells under aggregation. This is elegantly demonstrated by the tendency of the above systems to exhibit "blow-up" behaviour, in which aggregations of infinite cell density form. To develop a more realistic model for cell behaviour a model for chemotaxis was proposed by Painter and Hillen (2003), Hillen and Painter (2001). Their model incorporated the idea of "limited-space", as discussed above but within the context of a single-cell population. The inhibition of movement at higher densities prevented the "blow-up" behaviour observed in previous models and smooth aggregations developed (cf. Fig. 4(a) vs. (b)).

As our second example, we consider the situation where the tissue comprise two cell-types, $u$ and $v$, of which at least one is chemotactically stimulated by gradients of a chemical $c$. By assuming movement is influenced via combination of local, neighbour and gradient rules, we can derive the following two-cell model for chemotaxis using Eq. (10):

$$
\begin{aligned}
\frac{\partial u}{\partial t}= & \frac{\partial}{\partial x}\left[D_{u} g(w) \frac{\partial f(w) u}{\partial x}-D_{u} u f(w) \frac{\partial g(w)}{\partial x}\right. \\
& \left.-u g(w) f(w) \chi \frac{\partial c}{\partial x}\right], \\
\frac{\partial v}{\partial t}= & \frac{\partial}{\partial x}\left[D_{v} g(w) \frac{\partial f(w) v}{\partial x}-D_{v} v f(w) \frac{\partial g(w)}{\partial x}\right. \\
& \left.-v g(w) f(w) \phi \frac{\partial c}{\partial x}\right],
\end{aligned}
$$

$\frac{\partial c}{\partial t}=D_{c} \frac{\partial^{2} c}{\partial x^{2}}+\gamma_{1} u+\gamma_{2} v-\delta c$,

where $\gamma_{1}, \gamma_{2}, \delta, \chi, \phi, D_{u}, D_{v}$ and $D_{c}$ are all assumed constant and $w=u+v$. Initially we shall restrict our attention to the case where the cell-cell interaction occurs through "neighbour" rules only, and we therefore choose $g(w)=1-w / T$ and set $f(w) \equiv 1$. Note that the constant chemotactic sensitives above are derived by considering $\tau=\chi c$ or $\phi c$ for $u$ and $v$, respectively in Eq. (10). We assume zero-flux boundary conditions on the one-dimensional domain $[0, L]$.

The incorporation of two-cell populations allows us to study a number of relevant biological scenarios according to whether "autocrine"or "paracrine" behaviours are in operation. Here, the term autocrine refers to a situation where a single-cell type both secretes the 
chemical and migrates in response, while paracrine indicates a response in which one population secretes the chemical and the other migrates. The conditions under which we can expect aggregation can be calculated by performing a linear stability analysis on the above equations, and we refer to the appendix for details.

\subsection{Case 1. Autocrine $\left(\phi=0, \gamma_{2}=0\right)$}

We assume one cell both secretes and is attracted by the chemical ("auto-aggregation"): the second cell population is passive, and can only influences the dynamics via its "obstruction".

For comparison, we have included the equivalent independent movement scenario (achieved by additionally setting $g(w)=1$ above). This gives rise to the "classical" chemotaxis model, and for suitable parameters an initially homogeneous distribution of $u$ develops into a sharp cell aggregation. The $v$ population remains unperturbed from its homogeneous distribution, and has no effect either on the ability of patterns to form or the time-scale of patterning. Typical simulations are shown in Fig. 4(a).

Aggregation also occurs for the corresponding model incorporating the limited space interaction (we choose $g(w)=1-w / T)$. The condition for aggregation is given by the following equation (see appendix for details):

$\chi>\frac{\delta D_{u}}{\gamma_{1} u^{*}\left(1-u^{*} / T\right)}$.
Clearly, the passive population does not affect the conditions for spatial patterning to occur. This is shown by choosing three initial densities for the $v$ distribution. In the absence of $v$ cells [Fig. 4(b)] the $u$ cells aggregate into a smooth distribution, with the space limitation preventing the sharp aggregates characteristic of the linear case. This situation has been studied in detail in Painter and Hillen (2003), Hillen and Painter (2001). Increasing the density of $v$-cells does not affect the shape of the resulting aggregation of $u$-cells, [Fig. 4(c,d)], but it clearly changes the time for the aggregation to form. This follows intuitively from the ideas behind the limited space models.

\subsection{Case 2: Paracrine $\left(\chi=0, \gamma_{2}=0\right)$}

We now explore the paracrine case, in which $u$ secretes the chemical, and $v$ migrates in response to it. The comparable independent movement case is shown in Fig. 5(a): Pattern formation (see appendix for the analysis) is not possible in this model, since the secreting cell population remains homogeneously distributed throughout space.

The corresponding model incorporating interaction of the cells through space-limitation, however, does admit the possibility of pattern formation. This can intuitively be understood through the influence of the total cell density gradients on both populations, which results in the secreting cell population becoming inhomogeneously distributed. The linear stability analysis predicts that
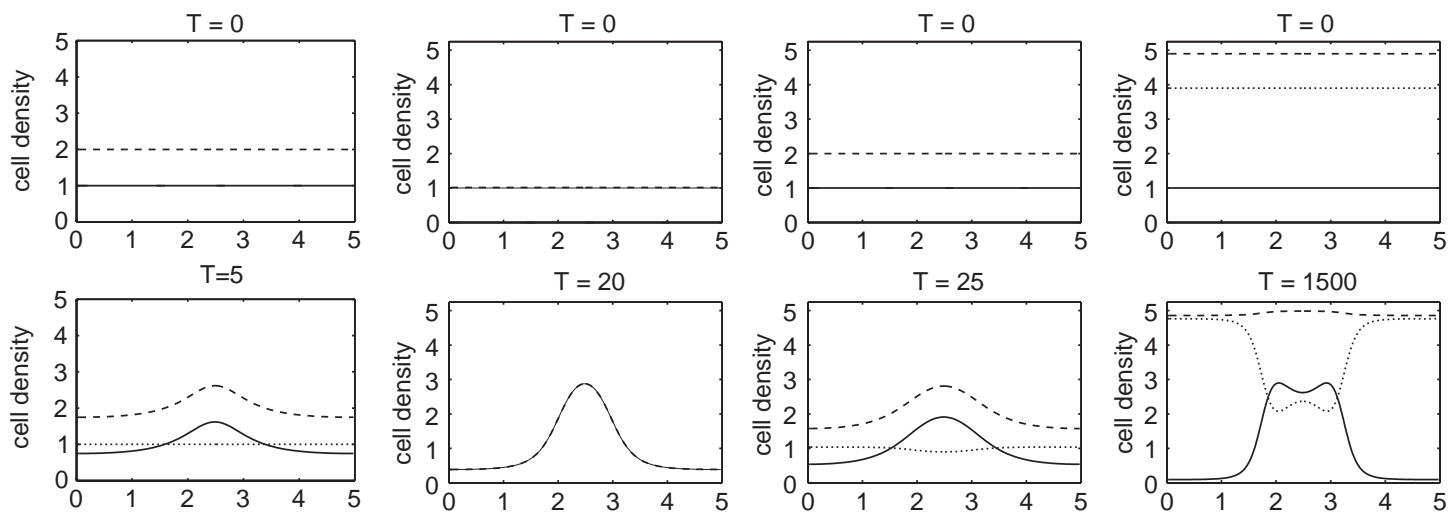

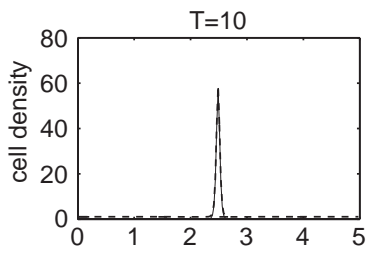

(a)

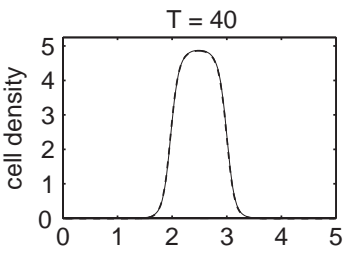

(b)

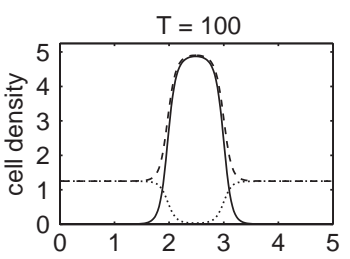

(c)

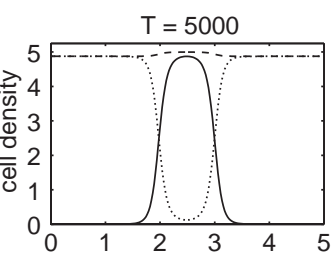

(d)

Fig. 4. Autocrine (auto-aggregation) case. (a) Two non-interacting cell populations (i.e. linear diffusion). (b)-(d) Interaction through spacelimitation for $T=5.0$ for (b) $v_{i}=0.0$, (c) $v_{i}=1.0$, (d) $v_{i}=3.9$. Other parameters are $\chi=1.0, \gamma_{1}=\delta=D_{c}=1.0, \gamma_{2}=\phi=0.0, D_{u}=D_{v}=0.1$ on a domain of $L=5$. Initial conditions are given by $u(x, 0)=1, v(x, 0)=v_{i}$ and $c(x, 0)=1.0-0.01 \cos (2 \pi x / L)$. Bold dashed line $=$ total cell density, solid line $=u$ density, dotted line $=v$ density. 

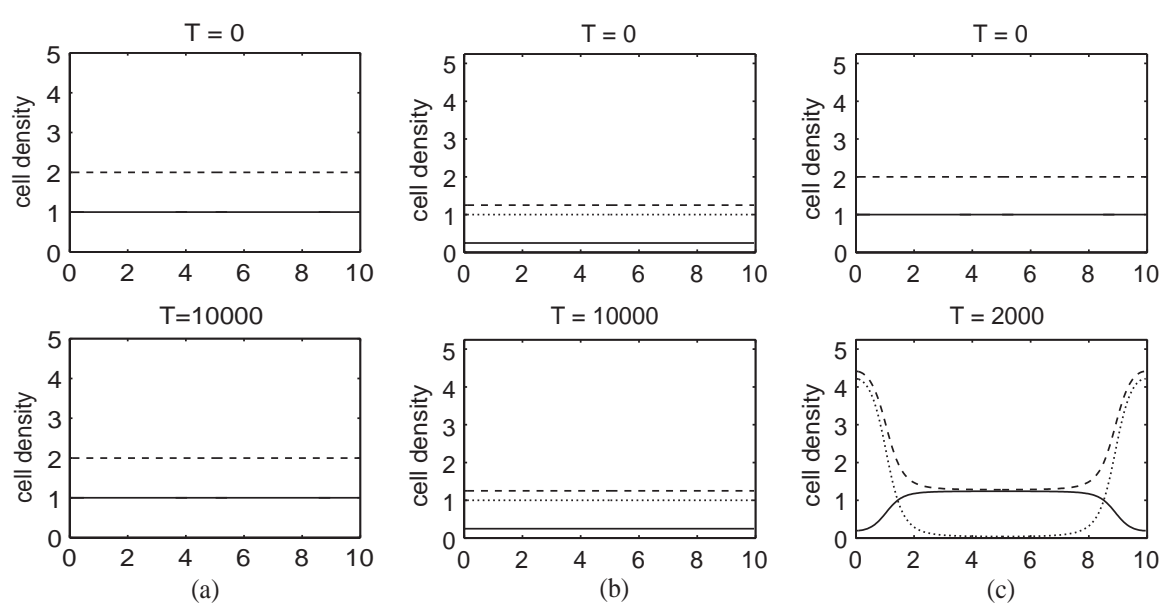

Fig. 5. Paracrine case. (a) Two non-interacting cell populations (i.e. linear diffusion). (b), (c) Interaction through space-limitation for $T=5.0$ for (a) $u_{i}=0.25$, (b) $u_{i}=1.0$. Other parameters are given by $\phi=-1.0, \gamma_{1}=\delta=D_{c}=1.0, \gamma_{2}=\chi=0.0, T=5.0 D_{u}=D_{v}=0.1$ on a domain of $L=10$. Initial conditions are given by $v(x, 0)=1, u(x, 0)=u_{i}$ and $c(x, 0)=1.0-0.1 \cos (2 \pi x / L)$. Bold dashed line $=$ total cell density, solid line $=u$ density, dotted line $=v$ density.

aggregation occurs under the following condition:

$\phi<-\frac{T \delta D_{v}}{u^{*} v^{*} \gamma_{1}}$.

Thus, pattern formation is only possible for a chemorepulsion response to the chemical. The density of the non-chemotacting population is crucial, and at low densities no aggregation is possible. Typical simulations at different densities of the non-chemotacting population $(u)$ are shown in Fig. 5(b), (c).

\subsection{Case 3. Autocrine + paracrine $\left(\gamma_{2}=0\right)$}

Finally, we consider a combined autocrine and paracrine response. Here the instability condition is

$D_{v}\left(1-u^{*} / T\right) \chi-\frac{D_{u} v^{*}}{T} \phi>\frac{\delta D_{u} D_{v}}{u^{*} \gamma_{1}}$.

The sign structure of $\chi-\phi$ influences the type of pattern that develops. If both cells are attracted by the chemical, the autocrine attraction $(\chi)$ must be sufficiently large such that these cells can "overcome" the paracrine population and aggregation take place. The resulting patterns take the form shown in Fig. 6(a), with the autocrine cells forming the aggregation core, and the paracrine cells confined mainly to the boundary.

For the autocrine population attracted $(\chi>0)$ and the paracrine population repelled $(\phi<0)$, the total cell density has multiple peaks, with attractant cells forming a core aggregation in the centre of the domain, and the $v$ cells repelled away to form aggregations at the chemical minima.

Patterning can even occur when the autocrine population is repelled by the chemical gradients $(\chi<0)$, provided that the paracrine cells show sufficiently strong chemorepulsion. A typical pattern is shown in Fig. 6(c).

\subsection{Inclusion of other total density effects}

In the above, the only tissue influence on movement resulted from space limitation. It is, of course, highly likely that other effects may occur at higher densities. For example, highly motile cells may force other cells out of the way as they pull themselves through the tissue environment. We incorporate such an effect phenomenologically by also including a "population-pressure" term in which a cell increases its tendency to move from a site as the density increases. Thus, we now additionally assume $f$ to be an increasing function of the total cell density, in addition to the previously chosen forms of $g$, $\tau$ in Eqs. (13).

As a test scenario, we consider the same generic case as illustrated in Fig. 6(a), where both cells are chemotactically attracted by gradients in the chemical concentration (though one cell shows decreased sensitivity). A choice of linear $f$ does not significantly change the behaviour, Fig. 7(a). However, it may be more appropriate to consider a form for $f$ such that it has little effect at low densities (i.e. when cells are widely spread out), but a strong effect when they are closely packed. For such forms, the results show a similar behaviour, but now the cells which have less sensitivity are "pushed" from the core of the aggregation to the periphery.

\section{Discussion}

Continuum models provide a convenient tool for describing the movements of populations. While detailed modelling of the movement in a tissue environment must incorporate a mechanical description of the 

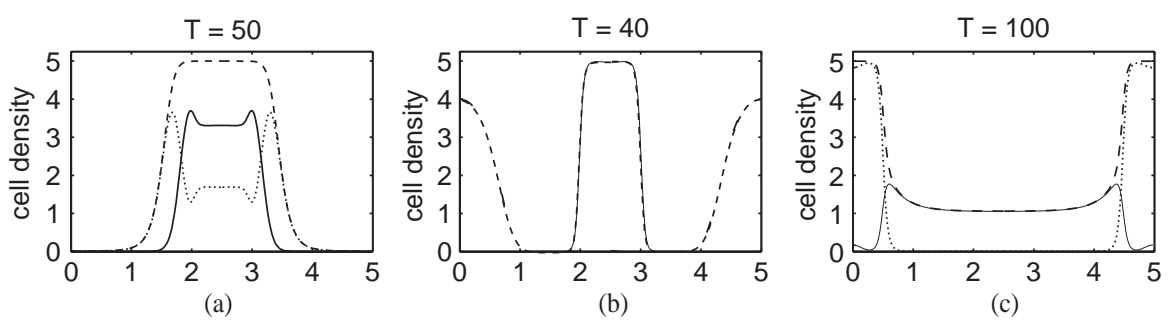

Fig. 6. Steady-state aggregations for two chemotacting populations (a) $\chi=2.0, \phi=1.0$ (b) $\chi=2.0, \phi=-2.0$ (c) $\chi=-1.0, \phi=-8.0$. Other parameters are given by $\gamma_{1}=\delta=D_{c}=1.0, \gamma_{2}=0.0, T=5.0 D_{u}=D_{v}=0.1$ on a domain of $L=5$, with initial conditions $u(x, 0)=1, v(x, 0)=1.0$ and $c(x, 0)=1.0-0.01 \cos (2 \pi x / L)$. Bold dashed line $=$ total cell density, solid line $=u$ density, dotted line $=v$ density.

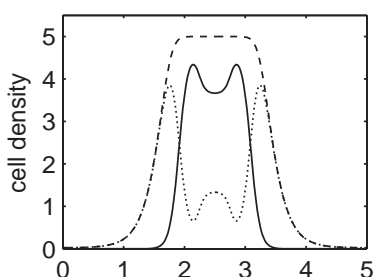

(a)

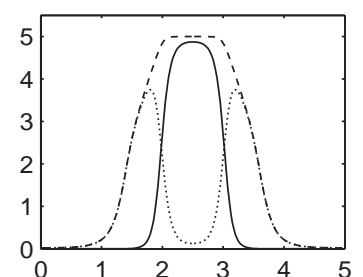

(b)

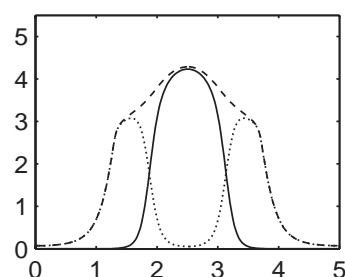

(c)

Fig. 7. Steady-state aggregations for two chemotacting populations including a "local pressure" effect. (a) $f(w)=a w($ b) $f(w)=1+\exp (2(w-3))$ (c) $f(w)=1+\exp (10(w-3))$ where $\chi=2.0, \phi=1.0, \gamma=\Delta=D_{c}=1.0, T=5.0 D_{u}=D_{v}=0.1$ on a domain of where $L=5$, with initial conditions $u(x, 0)=1, v(x, 0)=1.0 w_{0}=2$ and $c(x, 0)=1.0-0.01 \cos (2 \pi x / L)$.

various forces exerted by the cells, by adopting a phenomenological approach we have explored how interaction between different cell types may influence the macroscopic movements of cell populations. Our work here has focussed on scenarios for which the tissue exerts an equal effect on all cell populations. In reality, different populations vary in factors such as their adhesion to the matrix or the deformability of the membrane. Such variations may easily be incorporated into the framework here by choosing different functional forms/parameters in the movement equations.

The case studies in Sections 6 and 7 illustrate the importance of incorporating the interaction between cell populations into models for cell movement. In Section 6, this was demonstrated by the effect on the speed of wave propagation. In Section 7, a number of differences emerged in scenarios of aggregating cell populations. For example, in the "paracrine" problem, aggregation was only possible when interactions with another cell population were included.

The equations for competing cell populations given in Section 6 are highly generic, but the movement terms we have presented could be adapted to a range of more specific models. For example, competition between different subpopulations of cells is an integral part of the early growth of solid tumours. As well as the competition between tumour and normal cells, the cells within the tumour are a mixture of dividing and quiescent populations, which are self-organized into a characteristic layered structure. These various processes demand relatively sophisticated modelling of movement in interacting cell populations, and provide a potential case study for the ideas developed in this paper.

The simulation results of Section 7.4 bear similarities to processes of "cell sorting", in which an aggregate of initially mixed multiple cell types reorganize to form distinct regions, such that one-cell type forms the core of the aggregate while the other cells form the boundary. Explanations have primarily focussed on the "differential adhesion hypothesis" proposed by Steinberg (1970), in which differences in the levels of adhesion between the cells lead to the sorting. The results here suggest that the response to a diffusible chemical signal may also be a viable mechanism for certain sorting processes. In fact, such mechanisms has been shown to drive cell sorting of pre-stalk and pre-spore cells during Dictyostelium mound formation, (e.g. Weijer, 1999).

\section{Acknowledgements}

This work was supported by SHEFC Research Development Grant 107. JAS was also supported in part by an EPSRC Advanced Research Fellowship.

\section{Appendix A}

Here we detail the results of a linear stability analysis on the model for multi-cell chemotaxis, Eqs. (13), with 
$f(w) \equiv 1$. Assuming zero-flux boundary conditions on the one-dimensional domain $[0, L]$, then for suitable initial conditions (i.e. nonnegative) these equations have a single homogeneous steady state, $\left(u^{*}, v^{*}, c^{*}\right)$, where $u^{*}$ and $v^{*}$ are determined as $u^{*}=\int_{0}^{L} u(x, 0) \mathrm{d} x / L, v^{*}=$ $\int_{0}^{L} v(x, 0) \mathrm{d} x / L$ and $c^{*}=\left(\gamma_{1} u^{*}+\gamma_{2} v^{*}\right) / \delta$.

\section{A.1. Independent case}

If there is no direct interaction between the cells through the movement (i.e. $g(w)=1$ ), then a linearization of Eqs. (13). about the homogeneous steady state determines the following eigenvalue problem:

$$
\left|\begin{array}{ccc}
-\lambda-D_{u} k^{2} & 0 & \chi u^{*} k^{2} \\
0 & -\lambda-D_{v} k^{2} & \phi v^{*} k^{2} \\
\gamma_{1} & \gamma_{2} & -\lambda-k^{2} D_{c}-\delta
\end{array}\right|=0,
$$

where $\lambda$ is the (temporal) eigenvalue and $k$ is the wavenumber (or spatial eigenvalue) [e.g. see Murray (1993) for more details]. Thus the dispersion relation is a cubic polynomial of the form:

$\lambda^{3}+a\left(k^{2}\right) \lambda^{2}+b\left(k^{2}\right) \lambda+c\left(k^{2}\right)=0$.

Instability of the homogeneous solution, and thus the possibility of spatial pattern formation, occurs for $\mathbb{R}(\lambda)>0$. Since we can determine $a\left(k^{2}\right)>0$ for all positive $k^{2}$, then this can only occur if there exists a range of $k^{2}$ for which $b\left(k^{2}\right)<0$ or $c\left(k^{2}\right)<0$. We note that for $b\left(k^{2}\right)<0$ and $c\left(k^{2}\right)>0$ the eigenvalue may have imaginary components implying, at least initially, (temporally) oscillating solutions. For $c\left(k^{2}\right)<0$ we need $D_{u} D_{v} \delta<D_{u} \gamma_{2} \phi v^{*}+D_{v} \gamma_{1} \chi u^{*}$

and for $b\left(k^{2}\right)<0$ :

$D_{v} \delta+D_{u} \delta<\gamma_{2} \phi v^{*}+\gamma_{1} \chi u^{*}$.

In this paper, we concentrate on scenarios where at least one of $\gamma_{1}, \gamma_{2}, \chi$ or $\phi$ is zero. Under this constraint, it is easy to show that if $b\left(k^{2}\right)<0$ then we must also have $c\left(k^{2}\right)<0$. Thus the condition for instability of the homogeneous solution is given by Eq. (A.1) alone and positive eigenvalues are real.

Since all parameters are positive, it can be seen immediately from the above that aggregation is only possible if there is at least one autocrine response (i.e. $\gamma_{2}$ and $\phi$ non-zero or $\gamma_{1}$ and $\chi$ non-zero) - this corresponds to Cases 1 and 3 in Section 6. For the pure paracrine problem, (e.g. $\gamma_{1}$ and $\phi$ non-zero, $\gamma_{2}=\chi=0$ ), then the linear analysis does not predict spatial patterning.

\section{A.2. Interaction through neighbour rules}

A similar analysis can be performed for the case where the cells directly influence each others movement. Here we set $g(w)=1-w / T$ for $w=u+v$, and stipulate that $u(x, 0)+v(x, 0) \leqslant T$ (thus $w^{*}=u^{*}+v^{*} \leqslant T$ ). By linearization about the homogeneous steady state, we now yield the following eigenvalue problem:

$$
\left|\begin{array}{ccc}
-\lambda-k^{2}\left(D_{u} \frac{\left(1-v^{*}\right)}{T}\right) & -\frac{D_{u} u^{*} k^{2}}{T} & \chi u^{*} \frac{\left(1-w^{*}\right) k^{2}}{T} \\
-\frac{D_{v} v^{*} k^{2}}{T} & -\lambda-k^{2}\left(D_{v} \frac{\left(1-u^{*}\right)}{T}\right) & \phi v^{*} \frac{\left(1-w^{*}\right) k^{2}}{T} \\
\gamma_{1} & \gamma_{2} & -\lambda-k^{2} D_{c}-\delta
\end{array}\right|=0 .
$$

Again, we yield a cubic dispersion relation, and the conditions for $c\left(k^{2}\right)<0$ and $b\left(k^{2}\right)<0$ are, respectively:

$$
\begin{aligned}
& \frac{\chi u^{*}}{D_{u}}\left(\gamma_{1} T-v^{*} \gamma_{2}-u^{*} \gamma_{1}\right) \\
& \quad+\frac{\phi v^{*}}{D_{v}}\left(\gamma_{2} T-v^{*} \gamma_{2}-u^{*} \gamma_{1}\right)>T \delta
\end{aligned}
$$

and

$\gamma_{1} \chi u^{*}+\gamma_{2} \phi v^{*}>\frac{D_{u} \delta\left(1-v^{*} / T\right)+D_{v} \delta\left(1-u^{*} / T\right)}{\left(1-\left(u^{*}+v^{*}\right) / T\right)}$.

Again we concentrate on the conditions for the two main scenarios in Section 6. For the pure paracrine problem (Case 2), it is clear that Eq. (A.3) cannot be satisfied. Thus stability can only be lost through condition (A.2) being satisfied.

For the autocrine case (Case 1) we set $\phi=\gamma_{2}=0$. Eqs. (A.2) and (A.3) reduce to

$$
\chi u^{*} \gamma_{1}\left(1-u^{*} / T\right)>D_{u} \delta
$$

and

$\chi u^{*} \gamma_{1}>\frac{D_{u} \delta\left(1-v^{*} / T\right)+D_{v} \delta\left(1-u^{*} / T\right)}{1-\left(u^{*}+v^{*}\right) / T}$.

By multiplying through by $1-\left(u^{*}+v^{*}\right) / T$ in the latter it is easy to demonstrate that if the second condition holds, then so does the former. Thus, once again we do not expect any temporally oscillating solutions to the linearized system and we can reduce our stability criteria to the single condition.

In the scenario where only one of $\phi, \chi, \gamma_{1}$ or $\gamma_{2}$ is zero, then it also possible to determine parameter values such that $b\left(k^{2}\right)<0$ while $c\left(k^{2}\right)>0$. In such scenarios, we may have imaginary eigenvalues, leading to the possibility of temporally oscillating patterns.

\section{References}

Bottino, D.C., Fauci, L.J., 1998. A computational model of ameboid deformation and locomotion. Eur. Biophys. J. Biophys. 27 (5), $532-539$.

Bottino, D., Mogilner, A., Roberts, T., Stewart, M., Oster, G., 2002. How nematode sperm crawl. J. Cell Sci. 115 (2), 367-384.

Chaplain, M.A.J., Stuart, A.M., 1993. A model mechanism for the chemotactic response of endothelial cells to tumor angiogenesis factor. IMA J. Math. Appl. Med. 10 (3), 149-168.

Dallon, J.C., Othmer, H.G., 1997. A discrete cell model with adaptive signalling for aggregation of Dictyostelium discoideum. Philos. Trans. R. Soc. B 352, 391-417. 
Gaffney, E.A., Maini, P.K., Sherratt, J.A., Tuft, S., 1999. The mathematical modelling of cell kinetics in corneal epithelial wound healing. J. Theor. Biol. 197, 15-40.

Hillen, T., 2002. Hyperbolic models for chemosensitive movement. Math. Models Methods Appl. Sci. 12 (7), 1007-1034.

Hillen, T., Painter, K.J., 2001. A parabolic model with bounded chemotaxis - prevention of overcrowding. Adv. Appl. Math. 26, 280-301.

Hillen, T., Stevens, A., 2000. Hyperbolic models for chemotaxis in 1-d. Nonlinear Anal.: Real World Appl. 1 (3), 409-433.

Höfer, T., Sherratt, J.A., Maini, P.K., 1995. Dictyostelium discoideum: cellular self-organisation in an excitable biological medium. Proc. R. Soc. Lond. B 259, 249-257.

Keller, E.F., Segel, L.A., 1970. Initiation of slide mold aggregation viewed as an instability. J. Theor. Biol. 26, 99415.

Keller, E.F., Segel, L.A., 1971. Model for chemotaxis. J. Theor. Biol. 30, 225-234.

Murray, J.D., 1993. Mathematical Biology. Springer, Berlin, Heidelberg, New York.

Oster, G., 1984. On the crawling of cells. J. Embryol. Exp. Morphol. 83, 329-364.

Oster, G., Perelson, A., 1985. Cell spreading and motility: a model lamellipod. J. Math. Biol. 21, 383-388.

Othmer, H.G., Stevens, A., 1997. Aggregation, blowup and collapse: the ABC's of generalized taxis. SIAM J. Appl. Math. 57, 1044-1081.

Othmer, H.G., Dunbar, S.R., Alt, W., 1988. Models of dispersal in biological systems. J. Math. Biol. 26, 263-298.
Painter, K.J., Hillen, T., 2003. Volume-filling and quorum-sensing in models for chemosensitive movement, submitted for publication.

Painter, K.J., Maini, P.K., Othmer, H.G., 2000. A chemotactic model for the advance and retreat of the primitive streak in avian development. Bull. Math. Biol. 62, 501-525.

Pettet, G.J., Byrne, H.M., Mcelwain, D.L.S., Norbury, J., 1996. A model of wound-healing angiogenesis in soft tissue. Math. Biosci. $136(1), 35-63$.

Schnitzer, M., Block, S.M., Berg, H.C., Purcell, E.M., 1990. Biology of the Chemotactic Response, Vol. 46, Strategies for Chemotaxis. Cambridge University Press, Cambridge, MA, pp. 15-34.

Sherratt, J.A., 2000. Wavefront propagation in a competition equation with a new motility term modelling contact inhibition between cell populations. Proc. R. Soc. Lond. A 456, 2365-2386.

Sherratt, J.A., Nowak, M.A., 1992. Oncogenes, anti-oncogenes and the immune response to cancer. Proc. R. Soc. Lond. B 248, 261-272.

Steinberg, M.S., 1970. Does differential adhesion govern self-assembly processes in histogenesis? Equilibrium configurations and the emergence of a hierarchy among populations of embryonic cells. J. Exp. Zool. 173, 395-434.

Tyson, R., Lubkin, S.R., Murray, J.D., 1999. A minimal mechanism for bacterial pattern formation. Proc. R. Soc. Lond. B. Biol. 266, 299-304.

Weijer, C.J., 1999. Morphogenetic cell movements in dictyostelium. Sem. Cell Dev. Biol. 10, 609-619. 